ISSN 2076-393X

www.mdpi.com/journal/vaccines

Editorial

\title{
Acknowledgement to Reviewers of Vaccines in 2013
}

\section{Vaccines Editorial Office}

MDPI AG, Klybeckstrasse 64, CH-4057 Basel, Switzerland

Published: 28 February 2014

The editors of Vaccines would like to express their sincere gratitude to the following reviewers for assessing manuscripts in 2013:

$\begin{array}{lll}\text { Abel, Kristina } & \text { De Val, Bernat Pérez } & \text { Isaguliants, Maria } \\ \text { Acedo, L. } & \text { Dean, Gregg } & \text { Iurescia, Sandra } \\ \text { Ackerman, Margaret E. } & \text { Dempsey, Amanda F. } & \text { Kim, Kyung-Hyo } \\ \text { Agadjanyan, Michael } & \text { Derdeyn, Cynthia A. } & \text { Knudsen, Maria L. } \\ \text { Agwale, Simon M. } & \text { DiClemente, Ralph J. } & \text { Kobinger, Gary } \\ \text { Akiyama, Shinichiro } & \text { Dolstra, Harry } & \text { Koblin, Beryl } \\ \text { Antony, Paul A. } & \text { Dory, Daniel } & \text { Koff, Wayne C. } \\ \text { Barin, Francis } & \text { Duerr, Ann } & \text { Kortekaas, J. } \\ \text { Baron-Epel, Orna } & \text { Evans, Claire F. } & \text { Lao, T.T. } \\ \text { Barry, Peter } & \text { Fast, Patricia } & \text { Lapatra, Scott } \\ \text { Beckett, Charmagne G. } & \text { Field, Mark C. } & \text { Lau, Chuen-Yen } \\ \text { Biacchesi, Stéphane } & \text { Flick, Ramon } & \text { Lavail, Katherine Hart } \\ \text { Bigey, Pascal } & \text { Forde, Jonathan } & \text { Law, Barbara } \\ \text { Bjorkman, Pamela } & \text { Fujihashi, Kohtaro } & \text { Lichterfeld, Mathias } \\ \text { Brumme, Zabrina L. } & \text { Fujii, Shin-ichiro } & \text { Lin, Rongtuan } \\ \text { Castro, José María } & \text { Fujita, Mitsugu } & \text { Long, Elisa F. } \\ \text { Chang, Yung-Fu } & \text { Galli, Laura } & \text { Magez, S. } \\ \text { Chaput, Nathalie } & \text { Gianella, Sara } & \text { Mak, Kwok Kei } \\ \text { Chiappori, Alberto A. } & \text { Glanz, Jason } & \text { Martin, Michael } \\ \text { Cichutek, Klaus } & \text { Hanley, Sharon J.B. } & \text { Marzetta, Carol A. } \\ \text { Clayton, Christine } & \text { Harashima, Hideyoshi } & \text { McCune, Joseph (Mike) } \\ \text { Clements, C. John } & \text { Hertz, Tomer } & \text { Mcmichael, Andrew J. } \\ \text { Cossarizza, Andrea } & \text { Huh, Jooryung } & \text { Mcshane, Helen } \\ \text { Cowan, Anne } & \text { Inchauspé, Geneviève } & \text { Metzger, David S. } \\ & & \end{array}$


Metzger, Dennis W.

Mitchison, Timothy J.

Mizel, Steven B.

Murtaugh, Michael

Nan, Xiaoli

Nelson, Jennifer Clark

Nemazee, David

Niidome, Takuro

Nowak, Glen

Palmu, Arto A.

Papadimitriuou, Konstantinos

Pedersen, Anders E.

Petrovsky, Nikolai

Preat, Véronique

Purcell, Damian

Pushko, Peter

Rappuoli, Rino

Reisinger, Stephanie J.

Rolland, Morgane

Roxanne, Clark

Rudolph, Carsten
Ruffin, Mack

Schneewind, Olaf

Schuitemaker, Hanneke

Seib, Katherine

Shapiro, Lawrence

Shattock, Robin

Sheets, Rebecca L.

Shih, Mei-chiung

Simard, Edgar

Simon, Raphael

Slack, Mary

Slavcev, Roderick

Smerdou, Christian

Smiley, Stephen

Soren, Karen

Spearman, Paul

Stansbury, James

Stone, Geoffrey W.

Sullender, Wayne

Swamy, Geeta

Tomaras, Georgia D.
Tonyia, Eaves-Pyles

Trottein, François

Tsui, Jennifer

Turner, Stephen

Ueberla, Klaus

Uzonna, Jude

Van Wagoner, Nicholas J.

Villalba, Martin

Vitral, Claudia Lamarca

Wang, Xin

Warfield, Kelly L.

Wicker, S.

Willemen, Y.

Williams, James A.

Wyatt, Richard T.

Yusim, Karina

Zakhartchouk, Alexander

Zhu, Xiaoping

(C) 2014 by the authors; licensee MDPI, Basel, Switzerland. This article is an open access article distributed under the terms and conditions of the Creative Commons Attribution license (http://creativecommons.org/licenses/by/3.0/). 